\title{
PADRÕES ESPACIAIS DA MORTALIDADE INFANTIL E INDICADORES SOCIOECONÔMICOS NO MUNICÍPIO DE ALFENAS-MG
}

\author{
Mariana Rangel Garcia ${ }^{1}$ \\ Denis de Oliveira Rodrigues ${ }^{2}$ \\ Daniel Hideki Bando 3
}

RESUMO: Trata-se de um estudo de base populacional com o objetivo de identificar os padrões espaciais da Taxa de Mortalidade Infantil (TMI) e possíveis associações com indicadores socioeconômicos e de saneamento básico em Alfenas. Dados de estatísticas vitais foram obtidos da Secretaria Municipal de Saúde de Alfenas (2006 a 2018). E os indicadores socioeconômicos e de saneamento básico extraídos do censo demográfico (2010). A análise espacial dos indicadores foi realizada por mapas temáticos coropléticos. A TMI no período de estudo foi de 13,3 óbitos por mil nascidos vivos. As regiões Oeste 2 e Sul apresentaram as maiores taxas, (15,0 e 14,5 por mil nascidos, respectivamente) e sugerem associação direta com os "domicílios com esgoto a céu aberto" concentrados ao sul. Áreas na região Oeste 2, próximo ao bairro Distrito Industrial, também apresentaram percentuais relevantes de domicílios com "esgoto a céu aberto", "sem banheiro e com banheiro/esgotamento de fossa séptica", "com lixo acumulado nos logradouros", "com poço ou nascente na propriedade" e sinalizam associação direta com a TMI da região. As regiões Central, Sudeste e Leste mostraram-se com melhores condições relacionadas ao saneamento básico, em contraposição com as áreas periféricas.

PALAVRAS-CHAVE: geografia da saúde; mapa temático; regiões; análise espacial; AlfenasMG

\section{SPATIAL PATTERNS OF INFANT MORTALITY AND SOCIO-ECONOMIC INDICATORS IN THE MUNICIPALITY OF ALFENAS (MG)}

ABSTRACT: This is a population-based study with the objective of identifying the spatial patterns of the Infant Mortality Rate (IMR) and possible associations with socioeconomic and basic sanitation indicators in Alfenas. Vital statistics data were obtained from the Municipal Health Department of Alfenas (2006 to 2018). And the socioeconomic and

\footnotetext{
1 Licenciada em Geografia pela Universidade Federal de Alfenas; mariana.rangel@sou.unifalmg.edu.br

2 Vigilância Epidemiológica - Secretaria Municipal de Saúde de Alfenas; catoper@gmail.com

${ }^{3}$ Orientador e Docente de Geografia da Universidade Federal de Alfenas; daniel.bando@unifalmg.edu.br
} 
GARCIA, M. R; RODRIGUES, D. O; BANDO, D. H. Padrões espaciais de mortalidade infantil...

sanitation indicators extracted from the demographic census (2010). The spatial analysis of the indicators was carried out using choropleth thematic maps. The IMR during the study period was 13.3 deaths per thousand live births. The West 2 and South regions had the highest rates (15.0 and 14.5 per thousand births, respectively) and suggest a direct association with "households with open sewage" concentrated in the south. Areas in the West 2 region, close to the Distrito Industrial, also presented relevant percentages of households with "open sewer", "without bathroom and with bathroom/septic tank drainage", "with garbage accumulated in public places", "with well or spring on the property" and signal direct association with the IMR in the region. The Central, Southeast and East regions showed better conditions related to basic sanitation, in contrast to the peripheral areas.

KEYWORDS: health geography; thematic maps; regions; spatial analysis; Alfenas-MG

\section{PATRONES ESPACIALES DE MORTALIDAD INFANTIL E INDICADORES SOCIOECONÓMICOS EN EL MUNICIPIO DE ALFENAS (MG)}

RESUMEN: Se trata de un estudio de base poblacional con el objetivo de identificar los patrones espaciales de la Tasa de Mortalidad Infantil (TMI) y posibles asociaciones con indicadores socioeconómicos y de saneamiento básico en Alfenas. Los datos de las estadísticas vitales se obtuvieron del Departamento Municipal de Salud de Alfenas (2006 a 2018). Y los indicadores socioeconómicos y de saneamiento extraídos del censo demográfico (2010). El análisis espacial de los indicadores se realizó mediante mapas temáticos de coropletas. La TMI durante el período de estudio fue de 13,3 muertes por cada mil nacidos vivos. Las regiones Oeste 2 y Sur tuvieron las tasas más altas (15.0 y 14.5 por mil nacimientos, respectivamente) y sugieren una asociación directa con “hogares con alcantarillado abierto" concentrados en el sur. Áreas de la región Oeste 2, cercanas al Distrito Industrial, también presentaron porcentajes relevantes de hogares con "alcantarillado abierto", "sin baño y con baño / drenaje de fosa séptica", "con basura acumulada en lugares públicos", "con pozo o brotar en la propiedad "y señalar asociación directa con el TMI en la región. Las regiones Centro, Sudeste y Este mostraron mejores condiciones relacionadas con el saneamiento básico, en contraste con las áreas periféricas.

PALABRAS CLAVE: geografía de la salud; mapas temáticos; regiones; análisis espacial; Alfenas-MG

INTRODUÇÃO

A Taxa de Mortalidade Infantil (TMI) é um indicador que reflete não só as condições de saúde de crianças com idade até um ano, como também às 
GARCIA, M. R; RODRIGUES, D. O; BANDO, D. H. Padrões espaciais de mortalidade infantil...

condições sociais, econômicas e ambientais (BANDO, 2018; HELLER, 1997; TEIXEIRA et al., 2012). A mortalidade infantil apresenta profundas variações nos países, sendo que a média global em 2019 foi de 28,2 óbitos infantis por 1000 nascidos vivos (OMS, 2021). Em países como Finlândia e Japão a TMI foi inferior a 2 óbitos infantis por 1000 nascidos, já em países do continente Africano como Nigéria e Serra Leoa, as TMI foram superiores a 74 óbitos infantis por 1000 nascidos. No Brasil a TMI em 2019 foi de 12,5 óbitos infantis por 1000 nascidos (OMS, 2021).

A Taxa de Mortalidade Infantil (TMI) no Brasil tem diminuído progressivamente. Isso tem sido pensado mundialmente, e por diferentes órgãos governamentais. Os Objetivos de Desenvolvimento do Milênio (ODM), pautado pela Organização das Nações Unidas (ONU), em 2000, elencou oito objetivos, no qual o quarto se destacou por incentivar a redução da mortalidade infantil em 66\% no período de 1990 a 2015. Os mesmos, promoveram, às diversas, nações ações de assistência em saúde, estratégias políticas, acesso às boas condições de habitação e assistências à população (MOREIRA et al., 2012; LIMA et al., 2016; FARIA; SANTANA, 2016; KROPIWIEC et al., 2017; BRASIL, 2019). O Brasil alcançou a meta de redução taxa de mortalidade infantil já em 2011, atingindo a taxa de 15,3 óbitos (por mil nascidos vivos) (LIMA et al., 2016). Cabe destacar que a meta proposta tinha por objetivo atingir taxas inferiores a 15,7 óbitos por mil nascidos vivos, e que para a Organização Mundial da Saúde (OMS), a TMI aceitável é inferior a dois dígitos (MOREIRA et al., 2012). Considerando a definição a partir do Saúde Brasil (BRASIL, 2019), instrumento institucional produzido e organizado pela Secretaria de Vigilância em Saúde (SVS), a Taxa de Mortalidade Infantil (TMI) é calculada de acordo com a expressão:

Taxa de mortalidade infantil $=$ (número de óbitos de residentes com menos de 1 ano de idade $\div$ número de nascidos vivos de mães residentes) $\times 1.000$ 
GARCIA, M. R; RODRIGUES, D. O; BANDO, D. H. Padrões espaciais de mortalidade infantil...

A partir de estudos descritivos, o Saúde Brasil (2004; 2006; 2012; 2019) revela que a TMI teve declínio nas últimas sete décadas, com aproximadamente 146,6 óbitos (a cada mil nascidos vivos) em 1940; 47,5 em 1990; 26,8 em 2000; 16,2 em 2010, passando para 13,4 óbitos em 2017. A redução da taxa tem se demonstrado constante, no entanto ela não acontece de forma homogênea no país. As diferenças regionais a partir da TMI evidenciam a condição social, disponibilidade e qualidade dos recursos de cada localidade.

Estudos envolvendo diferentes escalas de análises da TMI mostram a forma heterogênea dos valores. O reflexo da desigualdade nas taxas pode aparecer nas regiões do país (ALMEIDA, 2016; BRASIL, 2019; SANTARELLI et al., 2019), nos estados e capitais (FARIA; SANTANA, 2016; BEZERRA FILHO et al., 2007; LIMA et al., 2016; CARVALHO et al., 2015) e nos municípios (KROPIWIEC et al., 2017; ALMEIDA, 2016; ). Dessa maneira, Holcman et al (2004) evidencia que dentro do território brasileiro existem níveis diferentes de mortalidade infantil. Em 2017 as maiores TMI registradas são de estados localizados na região Norte e Nordeste: Amapá (23,0 por mil nascidos vivos), Roraima $(19,8)$, Amazonas $(18,8)$, Piauí $(18,5)$ e Maranhão $(17,4)$. Em contraste com a região Sul, onde todos os estados tiveram as menores taxas do país, Santa Catarina $(9,9)$, Rio Grande do Sul $(10,1)$ e Paraná $(10,4)$ (BRASIL, 2019). O contraste entre as TMI das regiões se vincula em diferentes fatores, como a subnotificação dos óbitos, condições socioeconômicas, acesso aos serviços de saúde, escolaridade materna, idade de gestação, assistência durante a gestação entre outros (DUARTE, 2007; SANTOS et al., 2010; MAIA et al., 2012).

No estado de Minas Gerais foram observadas variações espaciais da TMI. As análises de Faria e Santana (2016), para o período de 2003 a 2013, mostram que o estado apresentou taxa menor que a média brasileira em todo o período observado, com valores de 16,5 (por mil nascidos vivos) de 2003 a 2007, e 13,5 em 
GARCIA, M. R; RODRIGUES, D. O; BANDO, D. H. Padrões espaciais de mortalidade infantil...

2008 a 2012. No entanto, perceberam que em 12\% dos municípios houve aumento do indicador, principalmente ao norte e nordeste do mesmo. Os estudos de Faria e Santana (2016) e Moreia et al. (2012) também destacaram a importância de implementações de políticas públicas para redução de mortalidade infantil no estado. Entre elas pode-se considerar a expansão de serviços de atenção primária à saúde, a criação em 2003 do Programa de Redução da Mortalidade Infantil e Materna em Minas Gerais, e ações em conjunto com o Ministério da Saúde.

Na literatura, a TMI pode ser analisada por meio de associações com indicadores socioeconômicos e em diferentes unidades de análise, como municípios e bairros (COSTA et al., 2020; FARIA; SANTANA, 2016). Entre os indicadores, as condições de saneamento básico estão entre os determinantes sociais que podem refletir na TMI (KROPIWIEC et al., 2017). Ademais, resultam das condições de habitação, renda, educação, saúde da população e acesso a serviços (LIMA, 2016).

Em conformidade a isso, o uso de Sistema de Informação Geográfica (SIG) tem sido ressaltado no campo da saúde pública (MAGALHÃES et al., 2006), por contribuir na análise espacial, avaliação das situações de saúde, qualidade de vida e condições ambientais (BARCELLOS et al., 2002). Nesse contexto, o presente estudo analisou espacialmente a TMI do município de Alfenas-MG e investigou as associações com indicadores socioeconômicos e de saneamento básico a partir de um estudo epidemiológico de base populacional.

\section{ÁREA DE ESTUDO}

O município de Alfenas está localizado na parte Sul do estado de Minas Gerais (Figura 1B), sendo dividido em 115 setores censitários (Figura 1C), sendo 103 setores urbanos (Figura 1D).

A partir de 1960, com a instalação do reservatório de Furnas, houve 
GARCIA, M. R; RODRIGUES, D. O; BANDO, D. H. Padrões espaciais de mortalidade infantil...

deslocamento da população rural para a cidade (BRANQUINHO; SILVA, 2018). De acordo com o censo de 2010, o município contou uma população urbana de 81,86\% (IBGE, 2010). Atualmente, o espaço rural, em sua maioria, está pautado na produção cafeeira, que toma grande expressão na economia regional, e nos usos e ocupações da terra (ALVES, 2018). No contexto educacional abrange grandes universidades, a de ensino privado UNIFENAS, e de ensino público UNIFAL. Alfenas também é referência na área da saúde e se inclui na Região de Saúde Alfenas Machado, formada por 16 municípios.

O município de Alfenas liderou o ranking de sua microrregião em diversos indicadores, apresentou maior número de habitantes (80.494) e Índice de Desenvolvimento Humano Municipal (IDHM) $(0,761)$.

Sobre saneamento e infraestrutura obteve maior percentual de rede geral de esgoto (93,07\%), lixo coletado por serviço de limpeza $(95,06 \%)$ e rede geral de água (94,02\%) (Tabela 1 onde IDHM: Índice de Desenvolvimento Humano Municipal; TMI: Taxa de Mortalidade Infantil (por mil nascidos vivos) no período de 2001 2018). A TMI em Alfenas foi de 13,34 óbitos infantis por mil nascidos vivos, próxima da microrregião, estando abaixo da média do país (14,03 por mil nascidos no ano de 2018) (BRASIL, 2019).

Figura 1 - Município de Alfenas setor censitário (C) e setores censitários urbanos (D). 
GARCIA, M. R; RODRIGUES, D. O; BANDO, D. H. Padrões espaciais de mortalidade infantil...
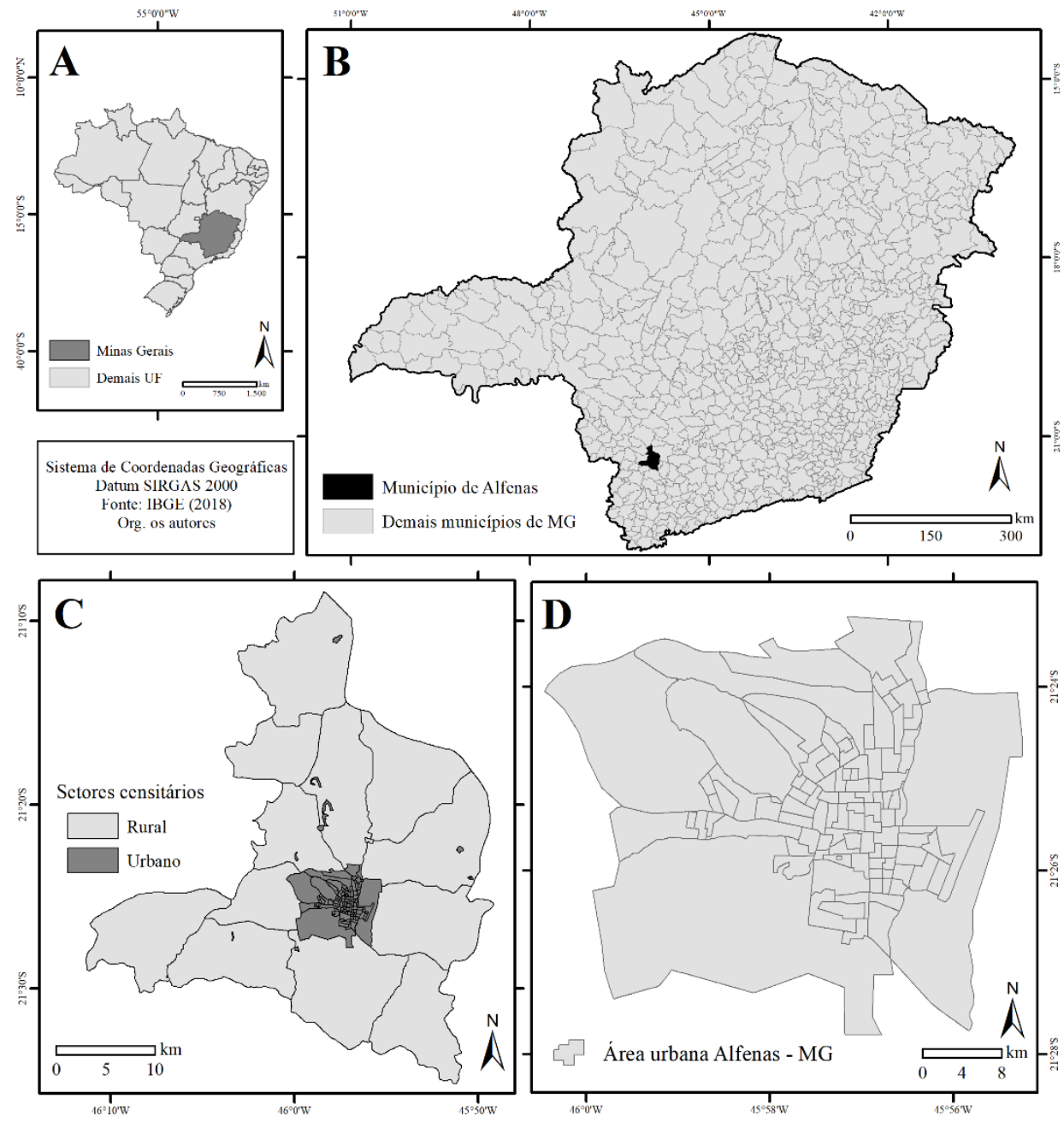
Tabela 1: Indicadores sociodemográficos dos municípios da Microrregião de Alfenas (MG)

\begin{tabular}{|c|c|c|c|c|c|c|}
\hline Municípios & $\begin{array}{l}\text { População } \\
\text { Estimada } \\
(2020)\end{array}$ & IDHM & TMI & $\begin{array}{c}\text { Rede Geral } \\
\text { de Esgoto } \\
(\%)\end{array}$ & $\begin{array}{l}\text { Lixo coletado por } \\
\text { serviço de limpeza } \\
\text { (\%) }\end{array}$ & $\begin{array}{c}\text { Rede } \\
\text { Geral de } \\
\text { água (\%) }\end{array}$ \\
\hline Alfenas & 80.494 & 0,761 & 13,34 & 93,07 & 95,06 & 94,02 \\
\hline Alterosa & 14.517 & 0,668 & 13,90 & 71,75 & 64,25 & 73,35 \\
\hline Areado & 15.181 & 0,727 & 13,73 & 83,22 & 83,82 & 82,91 \\
\hline $\begin{array}{l}\text { Carmo do } \\
\text { Rio Claro }\end{array}$ & 21.268 & 0,733 & 12,00 & 72,79 & 81,93 & 74,45 \\
\hline Carvalhópolis & 3.597 & 0,724 & 4,18 & 73,64 & 93,18 & 74,93 \\
\hline $\begin{array}{c}\text { Conceição da } \\
\text { Aparecida }\end{array}$ & 10.322 & 0,691 & 9,19 & 62,25 & 67,52 & 66,07 \\
\hline Divisa Nova & 6.025 & 0,670 & 15,72 & 75,42 & 82,3 & 80,55 \\
\hline Fama & 2376 & 0,717 & 10,44 & 49,33 & 73,87 & 65,69 \\
\hline Machado & 42.413 & 0,715 & 16,24 & 80,78 & 86,69 & 83,1 \\
\hline Paraguaçu & 21.605 & 0,715 & 13,13 & 80,35 & 83,57 & 80,73 \\
\hline Poço Fundo & 16.846 & 0,727 & 11,57 & 55,74 & 70,82 & 58,33 \\
\hline Serrania & 7.668 & 0,677 & 23,35 & 84,78 & 76,53 & 87,21 \\
\hline Total & 242.312 & 0,710 & 13,82 & 80,48 & 84,6 & 82,22 \\
\hline
\end{tabular}

Fonte: DATASUS, 2020; IBGE, 2010; 2020. Pnud Brasil, Ipea, FJP, 2020.

\section{MATERIAIS E MÉTODOS}

A base cartográfica vetorial do município de Alfenas por bairros foi extraída do projeto colaborativo Open Street Map (OSM, 2018) (Figura 2C).

Figura 2: Mapa da área de estudo - município de Alfenas por bairros e regiões. 
GARCIA, M. R; RODRIGUES, D. O; BANDO, D. H. Padrões espaciais de mortalidade infantil...

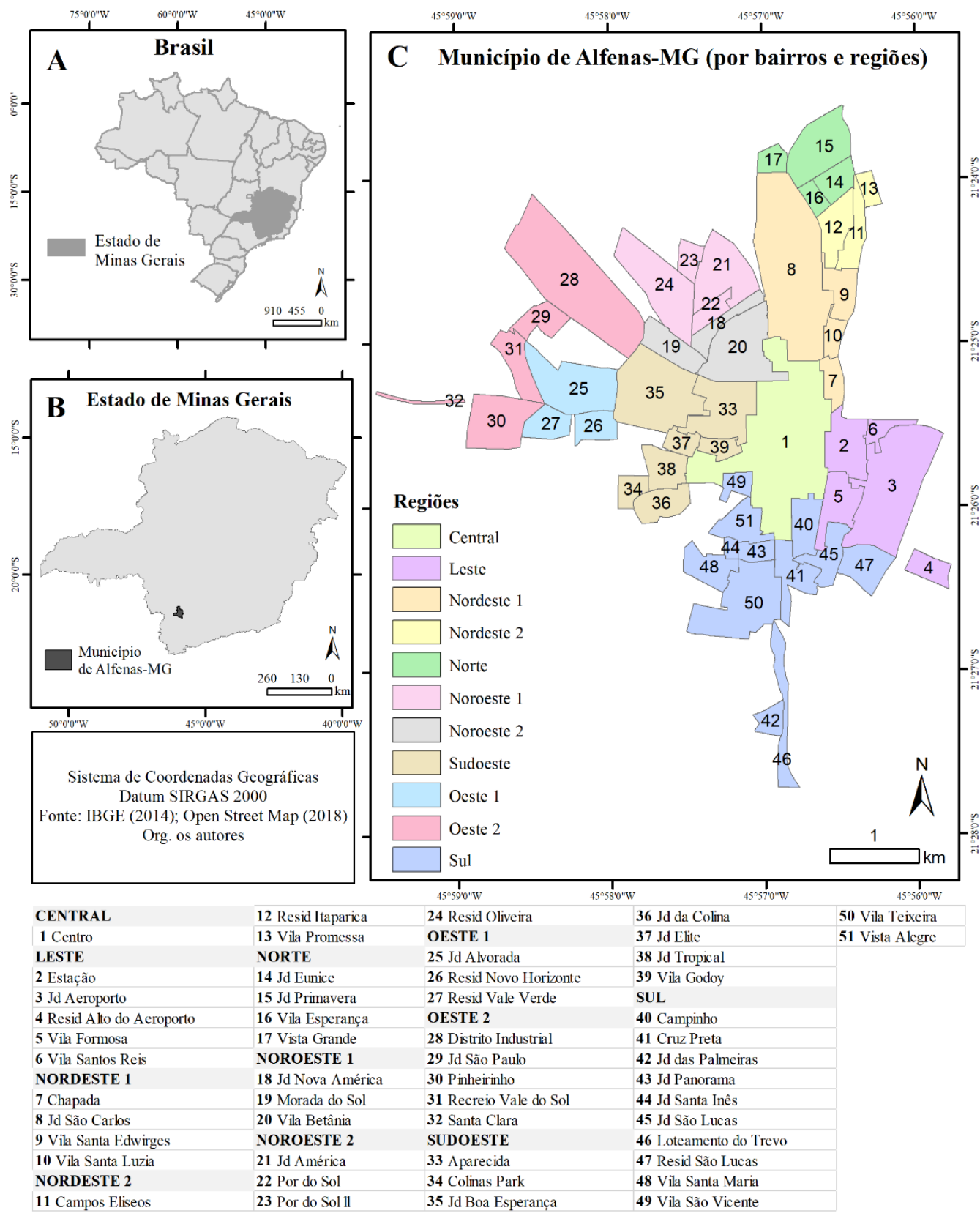

Trata-se de um estudo de base populacional com dados secundários, pautado na análise espacial da Taxa de Mortalidade Infantil (TMI) e de indicadores socioeconômicos e de saneamento básico no município de Alfenas-MG. A base de dados partiram da coleta das estatísticas vitais sendo: número de nascidos vivos e óbitos infantis, por bairro, obtidos diretamente da Secretaria Municipal de Saúde de Alfenas, no período de 2006 a 2018.

Os indicadores socioeconômicos e de saneamento foram obtidos a partir 
GARCIA, M. R; RODRIGUES, D. O; BANDO, D. H. Padrões espaciais de mortalidade infantil...

dos dados da Base de Informações do Censo Demográfico de 2010, relacionados ao entorno dos domicílios, por setor censitário (IBGE, 2010): domicílios com esgoto a céu aberto (\%); domicílios sem banheiro e domicílios com banheiro/esgotamento sanitário de fossa séptica (\%); domicílios com lixo acumulado nos logradouros (\%), domicílios com lixo depositado em caçamba de serviço de limpeza (\%); domicílios com poço ou nascente na propriedade (\%) e renda domiciliar per capita $(R \$)$.

Para elaboração dos mapas temáticos foi usado o Sistema de Informação Geográfica (SIG) ArcGIS 10.6 (ESRI, 2016), representados pelo método cartográfico coroplético (MARTINELLI, 1999), com intervalos classificados pelo método de quebra natural.

Inicialmente o objetivo do estudo foi utilizar a mesma unidade de análise para mapear todos indicadores, no caso o setor censitário, a menor unidade territorial utilizada pelo IBGE (2010). No entanto, a Secretaria Municipal de Saúde de Alfenas disponibilizou as estatísticas vitais por bairro, devido ao advento da Lei n 12.527/2011 (BRASIL, 2011) e a restrição de acesso à informação dos indivíduos. Portanto, a TMI foi mapeada por bairros e, os demais indicadores por setor censitário.

As análises envolveram três etapas. A primeira verificou a distribuição da TMI nos bairros. A TMI foi calculada pela razão entre o número de óbitos de menores de uma ano de idade, por mil nascidos vivos, na população residente em determinado espaço geográfico, no período considerado. Cabe destacar que numa análise preliminar, a TMI por bairros evidenciou distorções devido à instabilidade em pequenas áreas (SOUZA et al., 2007). A exemplo da TMI elevada no bairro Jardim Santa Inês (166,7 óbitos por mil nascidos vivos), no qual ocorreram seis nascidos vivos e apenas um óbito. Sendo assim, foi adotada a estratégia de agrupar os 51 bairros em 11 regiões: Central, Leste, Nordeste 1, Nordeste 2, Oeste 1, Oeste 2, Sudoeste e Sul (Figura 2C). O bairro Centro, por 
GARCIA, M. R; RODRIGUES, D. O; BANDO, D. H. Padrões espaciais de mortalidade infantil...

possuir o maior número de nascidos vivos (1491) foi denominado como região Central. Os demais bairros foram agrupados até atingir o número aproximado de mil nascidos vivos por região. A segunda etapa consistiu nas análises espaciais dos indicadores socioeconômicos e de saneamento. A terceira e última etapa, teve como propósito identificar possíveis associações entre a TMI e os indicadores socioeconômicos e de saneamento. Este estudo foi aprovado pelo Comitê de Ética e Pesquisa da Universidade Federal de Alfenas (CAAE 19453219.1.0000.5142).

\section{RESULTADOS E DISCUSSÕES}

No município de Alfenas, para o período de 2006 a 2018, ocorreram 143 óbitos infantis, e foram notificados 13.037 nascidos vivos, resultando numa TMI de 13,34 óbitos por mil nascidos vivos. A figura a seguir apresenta as TMI por região (Figura 3). 
GARCIA, M. R; RODRIGUES, D. O; BANDO, D. H. Padrões espaciais de mortalidade infantil...

Figura 3 - Taxa de Mortalidade Infantil (TMI) por região no município de Alfenas, 2006 a 2018

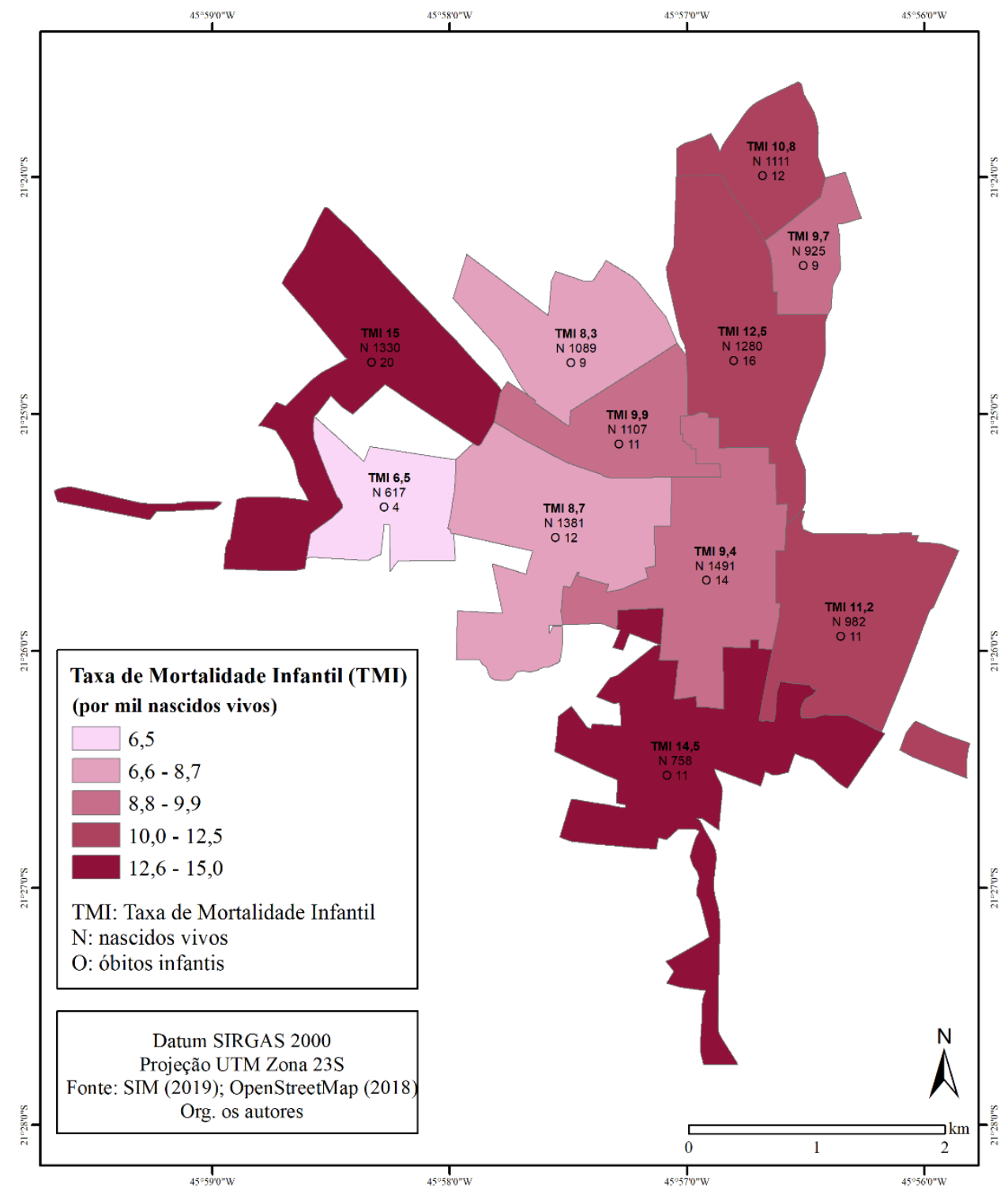

Vale destacar que o agrupamento das 11 regiões amenizou as distorções apresentadas nas TMI por bairros, dado que as maiores taxas variaram entre 12,6 a 15,0 óbitos por mil nascidos vivos. A região Oeste 2 apresentou a maior, com 15,0 óbitos por mil, seguida pela região Sul com 14,5 por mil. A maioria das regiões apresentaram a TMI na faixa de 8,8 a 12,5 por mil nascidos. A região Noroeste 2 e Sudoeste as TMI variaram entre 8,3 a 8,7 por mil nascidos, respectivamente. A região com a menor taxa foi a Oeste 1 com 6,5 por mil nascidos.

A Figura 4 apresenta os padrões espaciais dos indicadores socioeconômicos 
GARCIA, M. R; RODRIGUES, D. O; BANDO, D. H. Padrões espaciais de mortalidade infantil...

e de saneamento básico. A maioria dos setores $(75,7 \%)$ não apresentaram "domicílios com esgoto a céu aberto" (Figura 4A). Os setores equivalentes à região Sul, onde são de proximidade com os bairros Cruz Preta, Vila Teixeira e Vila Santa Maria, obtiveram os maiores percentuais variando de 13,2 a 20,3\%. O Plano Municipal de Saneamento Básico do município de Alfenas (ALFENAS, 2015) evidenciou o relato de cheiro de esgoto em todos os bairros o que, possivelmente, sugeriria falhas na prestação de serviços públicos e privados. No entanto informação se contrapõe com os dados do censo demográfico (Figura 4A). Os serviços relacionados ao saneamento básico do município são cumpridos pela Prefeitura Municipal de Alfenas (limpeza urbana), Companhia de Saneamento de Minas Gerais (COPASA) (fornecimento de água tratada, rede e coleta de esgoto) e a empresa Alfenas Ambiental (coleta domiciliar e comercial de resíduos sólidos) (ALFENAS, 2015).

Quanto ao indicador "domicílios sem banheiro e domicílios com banheiro/esgotamento sanitário por fossa séptica" nota-se que a maioria dos setores $(67,0 \%)$ não apresentavam essa condição (Figura 4B). Os setores que apresentaram percentuais diferentes de zero, atingiram o valor máximo de 2,6\%. Os resultados sugerem que os mesmos estão localizados nas áreas de periferia do município. Destaca-se o setor censitário a sudoeste que apresentou o maior percentual $(30,6 \%)$ e setores adjacentes que sugerem precarização dos serviços de rede geral de esgoto. A vulnerabilidade da cobertura desse serviço em áreas mais suscetíveis exprime riscos tanto a saúde como ao ambiente (ANDREAZZI et al., 2007). Além disso, áreas sem acesso a rede de esgoto podem destinar dejetos de forma irregular em corpos d'água, propiciando a poluição do mesmo e propagação de doenças. 
Figura 4 - Indicadores socioeconômicos e de saneamento por setores censitários em Alfenas-MG (2010)
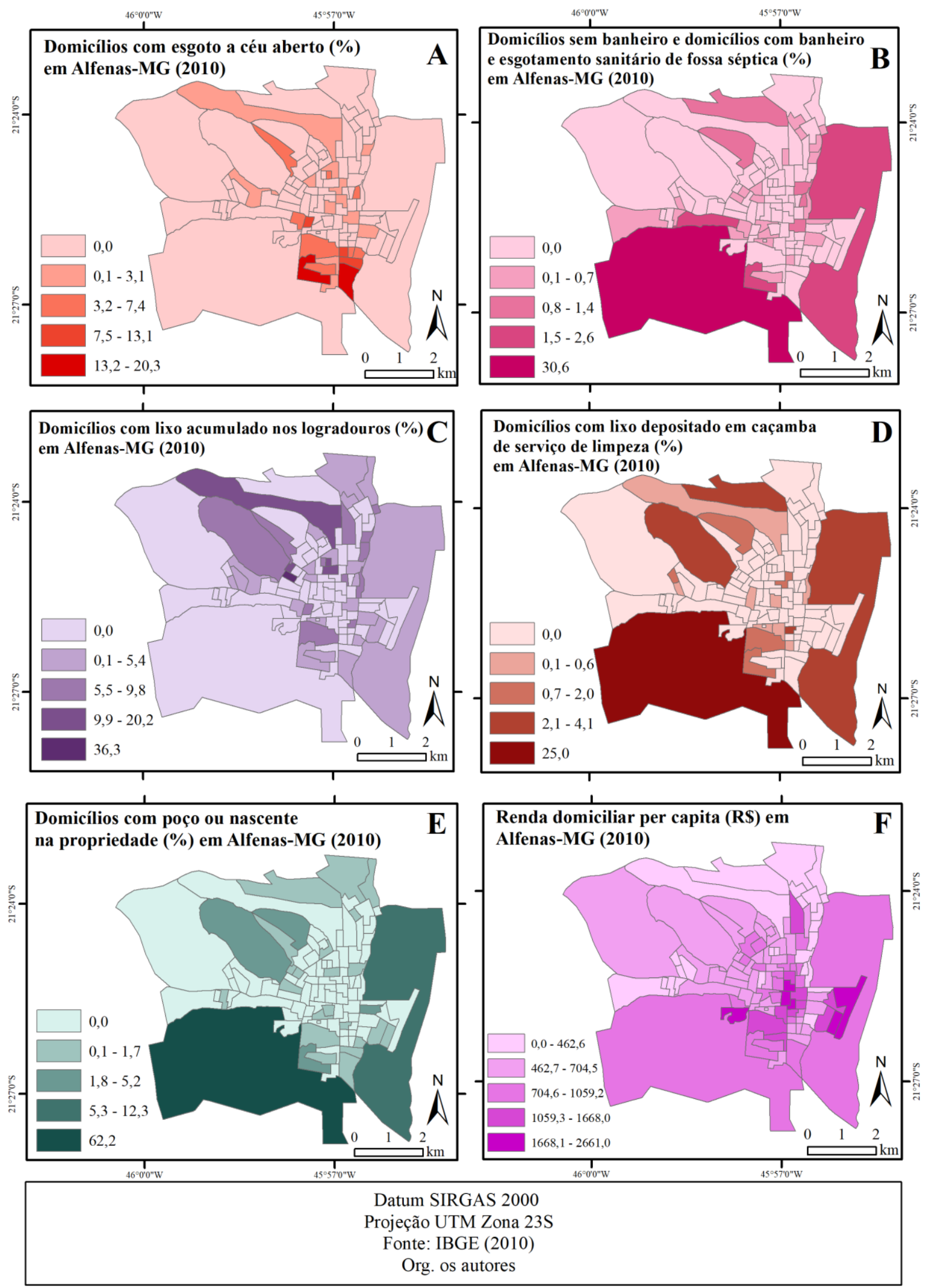
GARCIA, M. R; RODRIGUES, D. O; BANDO, D. H. Padrões espaciais de mortalidade infantil...

A maioria dos setores não apresentaram "domicílios com lixo acumulado nos logradouros" (62,1\%) (Figura 4C). Os setores que apresentaram consideráveis percentuais variaram entre 5,5 a 20,2\%, na área correspondente à região Oeste 2, na qual se localiza o bairro Distrito Industrial, possivelmente relacionado à atividade industrial. O percentual mais elevado (36,3\%) correspondeu a um setor próximo a essa área. Destaca-se também alguns setores da região Nordeste 1, com percentuais acima da média. A coleta de resíduos sólidos e lixos no município é terceirizado pela empresa Alfenas Ambiental. No Plano Municipal de Saneamento Básico (ALFENAS, 2015) consta que 71,3\% dos munícipes demonstram insatisfações com a realização da coleta de lixo. Ademais, 19,5\% notificaram que em suas ruas tinham casas ou lotes que funcionavam como depósito de lixo. Isso demonstra a problemática tanto na baixa consciência ambiental da população, como na efetividade na prestação de serviços.

Quanto ao indicador "domicílios com lixo depositado em caçamba de serviço de limpeza", verificou-se que não houve registro de ocorrência na maioria dos setores do município (82,5\%) (Figura 4D). Destacou-se novamente o setor na parte sudoeste que atingiu a máxima de $25,0 \%$. Foi percebido em algumas áreas relação direta desse indicador com "domicílios com lixo acumulado nos logradouros" (Figura 4C e 4D). A análise sugere que setores correspondentes às regiões Sul e Oeste 2 apresentaram percentuais relevantes em ambos os indicadores. Ou seja, levanta a hipótese de que o lixo depositado em caçamba pode influenciar o acúmulo de lixo no entorno.

Quanto ao abastecimento de água, nota-se que na maioria dos setores (72,8\%) não foram registradas ocorrências de "domicílios com poço ou nascente", ou seja, foram domicílios abastecidos por rede geral de distribuição de água (Figura 4E). Os setores que apresentaram valores significativos de “domicílios com poço ou nascente" variaram de 1,7 a 12,3\%, em setores periféricos. Destaca-se, mais uma vez, a máxima atingida no setor censitário a sudoeste (62,2\%). Alguns 
GARCIA, M. R; RODRIGUES, D. O; BANDO, D. H. Padrões espaciais de mortalidade infantil...

setores na região Sul, a leste do bairro Jardim Aeroporto e próximo ao Distrito Industrial também chamam a atenção. É possível notar semelhança na distribuição desse indicador com o "lixo acumulado" e "lixo depositado em caçamba" (Figura 4C e 4D). O Distrito Industrial tem avançado na instalação de indústrias (BRANQUINHO; SILVA, 2018), ou seja, demanda infraestrutura e condições que podem propiciar o acúmulo de lixo, despejos de dejetos de forma inadequada e esgoto a céu aberto.

Com relação a distribuição espacial da renda per capita, constatou-se que os setores próximos da região Central, região Leste (bairro Jd. Aeroporto) e uma porção da região Sudoeste (bairro Jd. da Colina e Colinas Park) apresentaram valores elevados, variando de $\mathrm{R} \$ 1668,1$ a $\mathrm{R} \$ 2661$,0 (Figura 4F). Em contraposição, os setores localizados na região Norte, Nordeste e Oeste 2 apresentaram renda domiliciar per capita de até $R \$ 462,6$.

No presente estudo, a TMI foi maior na região Oeste 2 e Sul de Alfenas e em parte coincide com o percentual de "domicílios com esgoto a céu aberto", concentrados no sul. O resultado sugere, portanto, associação direta entre os indicadores. Chamou a atenção também o setor censitário a sudoeste, que apresentou os maiores percentuais de "domicílios sem banheiro e domicílios com banheiro/esgotamento sanitário de fossa séptica" (30,6\%), "domicílios com lixo depositado em caçamba" (25,0\%), "domicílios com poço ou nascente" (62,0\%), e merece uma investigação com mais detalhes. Setores próximos à região Oeste 2 (Distrito Industrial) também apresentaram percentuais relevantes dos indicadores "domicílios com esgoto a céu aberto", "domicílios sem banheiro e com banheiro/esgotamento sanitário de fossa séptica", "domicílios com lixo acumulado nos logradouros", "domicílios com poço ou nascente" e sinalizam associação direta com a TMI da região Oeste 2. Cabe lembrar que a simples associação entre fenômenos não implica em relações de causalidade (SORRE, 1984), mas são evidências importantes que auxiliam a compreensão, e nos 
GARCIA, M. R; RODRIGUES, D. O; BANDO, D. H. Padrões espaciais de mortalidade infantil...

conduzem a novas perguntas e possíveis explicações.

Quanto aos padrões espaciais identificados no presente estudo, de acordo com Branquinho e Silva (2018), a condição das periferias no município de Alfenas obtém discordâncias, visto que nas mesmas chegam infraestruturas e serviços, no entanto, funcionam de forma precária. Contrapondo o que foi percebido nas regiões Central, parte da Sudoeste e Leste as quais possuem elevadas concentrações de renda, e melhores condições de infraestrutura e serviços. Os indicadores também sugerem que o ambiente (SUERTEGARAY, 2014) pode ser afetado, visto o percentual de domicílios com "esgoto a céu aberto", "lixo acumulado" e "poço ou nascente na propriedade". O planejamento integrado entre saúde, ambiente e sistemas de saneamento pode refletir diretamente na TMI, assim como na qualidade de vida da população (SOARES et al., 2002).

Foram encontrados estudos com dados secundários na literatura, em diversas escalas e locais do Brasil, que apontam para o mesmo sentido dos achados do presente estudo. Na revisão sistemática de Ferrari e Bertolozzi (2012) referente ao período de 2004 a 2009, demonstrou que as TMI são passíveis de redução, especialmente na população mais vulnerável socialmente, nas quais evidenciam precariedade em condições de moradia, educação, saneamento e acesso aos serviços de saúde. O estudo de Oliveira (2008) investigou os fatores socioeconômicos, demográficos e de atenção à saúde relacionados com as TMI nas microrregiões dos estados de Pernambuco, Minas Gerais e Rio de Janeiro. Os resultados puderam demonstrar que a falta do saneamento básico compunha o conjunto de variáveis que contribuíam para TMI elevadas. Bühler et al. (2014), realizou análise espacial de indicadores relacionados ao ambiente e saúde que pudessem ser condicionantes à mortalidade infantil por diarreias nas microrregiões brasileiras no ano de 2010. As microrregiões com elevadas TMI se situaram nas regiões Norte e Nordeste. Dentre os indicadores que demonstraram relação negativa com os óbitos infantis, foram o esgotamento sanitário e 
GARCIA, M. R; RODRIGUES, D. O; BANDO, D. H. Padrões espaciais de mortalidade infantil...

abastecimento de água. Apenas a Sudeste se associou positivamente com o indicador de extrema pobreza.

O presente estudo apresenta algumas limitações, sendo a primeira inerente ao desenho do estudo, onde foram utilizados dados agregados por setores censitários, bairros e regiões. Nesse tipo de estudo, o nível de associação entre os indicadores geralmente é mais tênue quando comparado a estudos a nível individual (ROTHMAN et al., 2011). No entanto, estudos desse tipo apresentam vantagens, como o baixo custo e facilidade na aquisição dos dados, por serem secundários. Além disso eles podem sugerir associações, gerar novas hipóteses e ser o ponto de partida para novos estudos (SZKLO; NIETO, 2007).

\section{CONSIDERAÇÕES FINAIS}

O presente estudo verificou os padrões espaciais da TMI e de indicadores socioeconômicos e de saneamento básico no município de Alfenas (MG). A TMI foi maior nas regiões Oeste 2 e Sul do município. Os resultados sugerem associação direta da TMI com o percentual de "domicílios com esgoto a céu aberto" concentrados ao sul. Destaca-se também alguns setores na região Oeste 2 (Distrito Industrial) que apresentaram percentuais relevantes de domicílios com "esgoto a céu aberto", com "lixo acumulado" e com "poço ou nascente na propriedade", possivelmente associadas com a TMI. Os indicadores revelaram maior vulnerabilidade social nas áreas periféricas de Alfenas. Portanto, os resultados podem auxiliar em melhorias de planejamento e gestão nos setores de saúde, saneamento básico e ambiente no município, assim como suporte para novas análises e hipóteses de investigação.

\section{REFERÊNCIAS BIBLIOGRÁFICAS}

ALMEIDA, W. S. Estimação da mortalidade infantil nos municípios brasileiros. Tese (Doutorado em Ciências) - Fundação Oswaldo Cruz, Rio de Janeiro, 2016. 
ANDREAZZI, M. A. R., BARCELLOS, C., HACON, S. Velhos indicadores para novos problemas: a relação entre saneamento e saúde. Revista Panam Salud Publica. v. 22, n. 3, p.211-217, 2007.

BANDO, D. H. Geografia da saúde mineira. In: FERREIRA, M. M.; VALE, A. R. L. Dinâmicas Geográficas no Sul de Minas Gerais. Curitiba, Appris, 2018, p. 49-78.

BARCELLOS, C. C.; SABROZA, P. C.; PEITER, P.; IÑIGUEZ, L. R. Organização espacial, saúde e qualidade de vida: análise espacial e uso de indicadores na avaliação de situações de saúde. Informe Epidemiológico do SUS, Brasília, v.11, n.3, p.129-138, 2002.

BRANQUINHO, E.; SILVA, L. S. A Reestruturação das cidades médias: o caso de Alfenas no Sul de Minas Gerais. In: FERREIRA, M. M.; VALE, A. R. L. Dinâmicas Geográficas no Sul de Minas Gerais. Curitiba, Editora Appris, 2018, p.79-106.

BRASIL, Lei $n^{\circ} 12.527$, de 18 de novembro de 2011. Regula o acesso a informações previstos no inciso XXXIII do art. $5^{\circ}$, no inciso II do $\S 3^{\circ}$ do art. 37 e no $\S 2^{\circ}$ do art. 216 da Constituição Federal. Brasília, 2011. Disponível em: http://www.planalto.gov.br/ccivil_03/_ato2011-2014/2011/lei/l12527.htm

BRASIL. Ministério da Saúde. Banco de dados do Sistema Único de Saúde DATASUS. Disponível em: http://www.datasus.gov.br/DATASUS

BRASIL. Ministério da Saúde. Secretaria de Vigilância em Saúde. Departamento de Análise de Situação de Saúde. Saúde Brasil 2004 - uma análise da situação de saúde / Ministério da Saúde, Secretaria de Vigilância em Saúde, Departamento de Análise de Situação de Saúde. - Brasília: Ministério da Saúde, 2004.

Saúde Brasil 2006: uma análise da situação de saúde no Brasil / Ministério da Saúde, Secretaria de Vigilância em Saúde, Departamento de Análise de Situação em Saúde. - Brasília: Ministério da Saúde, 2006.

Saúde Brasil 2011: uma análise da situação de saúde e a vigilância da saúde da mulher / Ministério da Saúde, Secretaria de Vigilância em Saúde, Departamento de Análise de Situação de Saúde. - Brasília: Editora do Ministério da Saúde, 2012.

BRASIL. Ministério da Saúde. Secretaria de Vigilância em Saúde. Departamento de Análise em Saúde e Vigilância de Doenças Não Transmissíveis. Saúde Brasil 2019 uma análise da situação de saúde com enfoque nas doenças imunopreveníveis e na imunização. Brasília: Ministério da Saúde. 2019. 
BÜLER, H. F.; IGNOTTI, E.; NEVES, S. M. A. S; HACON, S. S. Análise espacial de indicadores integrados determinantes da mortalidade por diarreia aguda em crianças menores de 1 ano em regiões geográficas. Ciência \& Saúde Coletiva, v.19, n.10, p.4131-4140, 2014.

CARVALHO, R. A. S.; SANTOS, V. S.; MELO, C. M.; GURGEL, R. Q.; OLIVEIRA, C. C. C. Desigualdades em saúde: condições de vida e mortalidade infantil em região do nordeste do Brasil. Revista de Saúde Pública, v.49, n.5, p.1-9, 2015.

COSTA, S. S.; HELLER, L.; BRANDÃO, C. C. S.; COLOSIMO, E. A. Indicadores epidemiológicos aplicáveis a estudos sobre a associação entre saneamento e saúde de base municipal. Engenharia Sanitária e Ambiental, v.10, n.2, p.118-127, 2005.

DUARTE, C. M. R. Reflexos das políticas de saúde sobre as tendências da mortalidade infantil no Brasil: revisão de literatura sobre a última década. Caderno de Saúde Pública, Rio de Janeiro, v.23, n.7, p.1511-1528, 2007.

FARIA, R.; SANTANA, P. Variações espaciais e desigualdades regionais no indicador de mortalidade infantil do estado de Minas Gerais, Brasil. Saúde e Sociedade, São Paulo, v.25, n.3, p.736-749, 2016.

FERRARI, R. A. P.; BERTOLOZZI, M. R. Mortalidade pós-neonatal no território brasileiro: uma revisão de literatura. Revista da Escola de Enfermagem da USP, São Paulo, v.5, n.46, p.1207-1214, 2012.

HELLER, L. Saneamento e Saúde. Organização Pan-Americana da Saúde/Organização Mundial da Saúde. Brasília, 1997.

IBGE. Instituto Brasileiro de Geografia e Estatística. Base de informações do Censo Demográfico 2010: Resultados do Universo por setor censitário. Ministério de Planejamento, Orçamento e Gestão. Rio de Janeiro, 2011.

IBGE. Instituto Brasileiro de Geografia e Estatística. Dados do Censo Demográfico. Disponível em: https://cidades.ibge.gov.br/. Acessado em: 06 de dezembro de 2020.

KROPIWIEC, M. V.; FRANCO, S. C.; AMARAL, A. R. Fatores associados à mortalidade infantil com índice de desenvolvimento humano elevado. Revista Paul Pediatr, v.35, n.4, p.391-398, 2017.

LIMA, S. C. Território e promoção da saúde: perspectivas para a atenção primária à saúde. Jundiaí - SP: Paco editorial, 2016. 
GARCIA, M. R; RODRIGUES, D. O; BANDO, D. H. Padrões espaciais de mortalidade infantil...

MAGALHÃES, M. A. F. M.; SANTOS, S. M.; GRACIE, R.; BARCELLOS, C. Sistemas de Informações Geográficas em saúde. In: BRASIL, Ministério da Saúde. Secretaria de Vigilância em Saúde. Fundação Oswaldo Cruz. Abordagens espaciais em na saúde pública. Brasília: Ministério da Saúde, 2006.

MAIA, L. T. S.; SOUZA, W. V.; MENDES, A. C. G. Diferenciais nos fatores de risco para a mortalidade infantil em cinco cidades brasileiras: um estudo de caso-controle com base no SIM e no SINASC. Caderno de Saúde Pública, Rio de Janeiro, v.28, n.11, p.2163-2176, p.2012.

MARTINELLI, M. As representações gráficas da Geografia: os mapas temáticos. 1999.

MOREIRA, L. M. C.; ALVES, C. R. L.; BELISÁRIO, S. A.; BUENO, M. C. Políticas públicas voltadas para a redução da mortalidade infantil: uma história de desafios. Revista Médica de Minas Gerais, v.22, p.48-55, 2012.

OLIVEIRA, S. M. M. C. Mortalidade Infantil e Saneamento Básico - ainda uma velha questão. In: XVI ENCONTRO NACIONAL DE ESTUDOS POPULACIONAIS, CaxambuMG, de 29 de setembro a 03 de outubro de 2008. Anais do XVI Encontro Nacional de Estudos Populacionais, p.1-21.

OMS. Organização Mundial da Saúde. Observatório Global de Saúde. Disponível em: https://www.who.int/data/gho/data/indicators/indicator-details/GHO/infantmortality-rate-(probability-of-dying-between-birth-and-age-1-per-1000-livebirths). Acessado em novembro 2021.

PLANO DIRETOR PARTICIPATIVO DE ALFENAS. Prefeitura Municipal de Alfenas. Lei n. 3.941 de 12 de dezembro de 2006.

PNUD.; IPEA.; FJP. Índice de Desenvolvimento Humano Municipal Brasileiro. Brasília, 2013.

ROTHMAN, K. J.; GREENLAND, S.; LASH, T. L. Tipos de estudos epidemiológicos. In: ROTHMAN, K. J.;GREENLAND, S. Epidemiologia Moderna. Porto Alegre: Artmed, 2011. cap. 6, p. 107-122.

SALTARELLI, R. M. F.; PRADO, R. R.; MONTEIRO, R. A.; MALTA, D. C. Tendência da mortalidade por causas evitáveis na infância: contribuições para a avaliação de desempenho dos serviços públicos de saúde da Região Sudeste do Brasil. Revista Brasileira de Epidemiologia, v.22, p.1-15, 2019.

SANTOS, H. G.; ANDRADE, S. M.; BIROLIM, M. M.; CARVALHO, W. O.; SILVA, A. M. R. 
GARCIA, M. R; RODRIGUES, D. O; BANDO, D. H. Padrões espaciais de mortalidade infantil...

Mortalidade infantil no Brasil: uma revisão de literatura antes e após a implantação do Sistema Único de Saúde. Pediatria, São Paulo, v.32, n.2, p.131-143, 2010.

SOARES, S.R.A.; BERNARDES, R.S.; CORDEIRO NETTO, O.M. Relações entre saneamento, saúde pública e meio ambiente: elementos para formulação de um modelo de planejamento em saneamento. Cadernos de Saúde Pública, Rio de Janeiro, v. 18, p. 1713-1724, 2002.

SORRE, M. Geografia. Coleção grandes cientistas sociais. São Paulo: Ática, 1984.

SOUZA, W. V.; CARVALHO, M. S.; CRUZ, O. G.; RAGONI, V. Análise Espacial de Dados de Áreas. In: BRASIL, Ministério da Saúde. Secretaria de Vigilância em Saúde. Fundação Oswaldo Cruz. et al (Ed.). Introdução à Estatística Espacial para a Saúde Pública. 1 ed. Brasília: Ministério da Saúde, v. 3, cap. 3, 2007.

SUERTEGARAY, D. M. A. Geografia e Ambiente: desafios ou novos olhares. Revista Mato-Grossense de Geografia, Cuiabá - v.17, n.1, p. 3-14, 2014.

SZKLO, M.; NIETO, F. J. Epidemiology: beyond the basics. Sudbury: Jones and Bartlett Publishers, 2007.

TEIXEIRA, J. C.; GOMES, M. H. R.; SOUZA, J. A. Associação entre cobertura por serviços de saneamento e indicadores epidemiológicos nos países da América Latina: estudo com dados secundários. Revista Panam Salud Publica. v.32, n.6, p.419-425, 2012. 